\title{
A NOVEL MEASURE FOR SYNCHRONY AND ITS APPLICATION TO NEURAL SIGNALS
}

\author{
Justin Dauwels, François Vialatte and Andrzej Cichocki \\ RIKEN Brain Science Institute, Saitama, Japan \\ email: justin@dauwels.com, \{fvialatte, cia\}@brain.riken.jp
}

\begin{abstract}
A novel measure to quantify the synchrony between two sparse binary strings is proposed, referred to as "stochastic event synchrony" (SES). It is computed by performing inference in a probabilistic model. SES can amongst other be used to detect synchrony in neural signals, in particular, spike trains (obtained from electrophysiological recordings) and EEG signals. It is demonstrated how SES can quantify the firing reliability of a neuron. It is also shown how SES can be used as a feature to detect Alzheimer's disease based on EEG signals.
\end{abstract}

Index Terms- Synchronization, Inference, Electrophysiology, Electroencephalography, Feature Extraction

\section{INTRODUCTION}

A sparse binary string is a sequence of bits in which the vast majority of elements are zero (see e.g., Fig. 1, top). Sparse binary sequences occur in many contexts, for example, in digital communications, applied information theory, digital circuit theory, image processing, and neuroscience. A sparse binary string can compactly be represented by an event string, which is the list of time instances at which the ones ("events") occur; for example, the event string corresponding to the sparse binary string at the top of Fig. 1 is given by $(2,8,10,27,29)$.

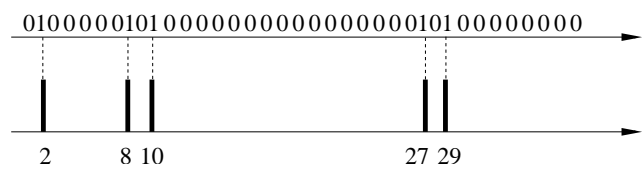

Fig. 1. A sparse binary sequence and its event string.

In this paper, we propose a novel measure to quantify the synchrony between two event strings, referred to as stochastic event synchrony (SES). We apply this synchrony measure in the context of neuroscience. Synchrony is an important topic in neuroscience. For instance, it is hotly debated whether the synchronous firing of neurons plays a role in cognition [1] and even in consciousness [2]. The synchronous firing paradigm has also attracted substantial attention in both the experimental (e.g., [3]) and the theoretical neuroscience literature (e.g., [4]). By means of SES, one can detect patterns of synchrony in recorded spike trains. Therefore, SES may be of interest to neuroscientists who wish to validate theoretical neural models that involve synchronous neural firing. As we will demonstrate, the proposed synchronization measure may also be used to quantify how reliably a single neuron fires when activated by a certain stimulus ("firing reliability") [7].
SES can also be applied in an entirely different context, i.e., for the detection of synchrony in multi-electrode EEG signals. This may surprise the reader, since EEG signals are continuous-valued signals, and therefore, they seem to have little in common with event strings. We will show, however, how event strings can be extracted from EEG signals. In the long term, the proposed method may be helpful to detect mental disorders such as Alzheimer's disease, since mental disorders are often associated with abnormal blood and neural activity flows, and changes in the synchrony of the activity of different brain regions (see, e.g., [5]). In this paper, we will present promising preliminary results on the detection of Alzheimer's disease from EEG signals based on SES.

This paper is organized as follows. In the next section, we introduce stochastic event synchrony (SES). In Section 3, we briefly outline two algorithms to compute the SES parameters for a given pair of sparse binary strings. In Section 4, we quantify the firing reliability of a single neuron by means of SES. In Section 5, we compute the SES parameters for multi-electrode EEG signals; we demonstrate how those parameters can be used as features to distinguish Alzheimer's disease patients from control patients. We offer some concluding remarks in Section 6.

\section{STOCHASTIC EVENT SYNCHRONY (SES)}

Let us consider the two event strings $X$ and $X^{\prime}$ in Fig. 2 (ignore the strings $Y$ and $Z$ for now). We wish to quantify to which extent $X$ and $X^{\prime}$ are synchronized. Intuitively speaking, two event strings can be considered as synchronous if they are identical apart from: (i) a time shift $\delta_{T}$; (ii) small deviations in the event occurrence times ("event timing jitter"); (iii) a few event insertions and deletions.

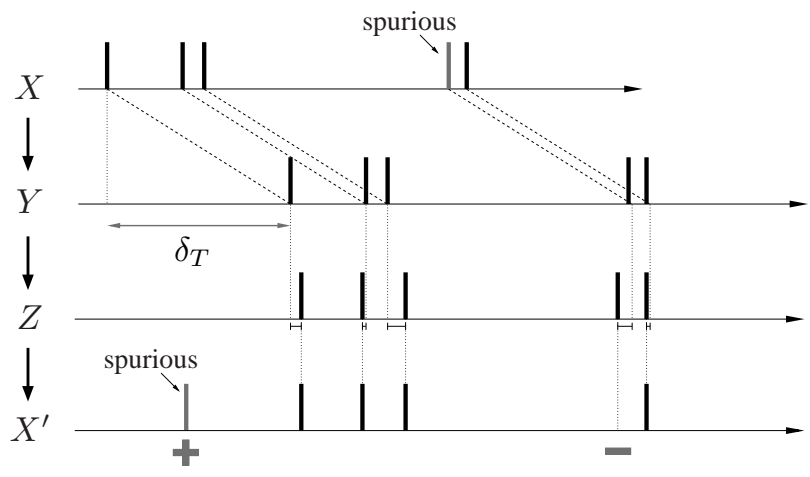

Fig. 2. Stochastic Event Synchrony. 
More precisely, for two event strings to be synchronous, the event timing jitter should be significantly smaller than the average inter-event time, and the number of deletions and insertions should comprise only a small fraction of the total number of events. This intuitive concept of synchrony is illustrated in Fig. 2. The event string $X^{\prime}$ is obtained from event string $X$ by successively shifting $X$ over $\delta_{T}$ (resulting in $Y$ ), slightly perturbing the event occurrence times (resulting in $Z$ ), and eventually, by adding (plus sign) and deleting (minus sign) events, resulting in $X^{\prime}$. Adding and deleting spikes in $Z$ leads to "spurious" events in $X$ and $X^{\prime}$ (see Fig. 2): a spurious event in $X$ is an event that cannot be paired with an event in $X^{\prime}$ and vice versa.

The above intuitive reasoning leads to our novel measure for synchrony between two event strings, i.e., stochastic event synchrony (SES); it is defined as the triplet $\left(\delta_{T}, \sigma_{T}, \rho_{\text {spur }}\right)$, where $\sigma_{T}$ is the standard deviation of the (event) timing jitter, and $\rho_{\text {spur }}$ is the percentage of spurious spikes

$$
\rho_{\text {spur }} \triangleq \frac{N_{\text {spur }}+N_{\text {spur }}^{\prime}}{N+N^{\prime}}
$$

with $N$ and $N^{\prime}$ the total number of events in $X$ and $X^{\prime}$ respectively, and $N_{\text {spur }}$ and $N_{\text {spur }}^{\prime}$ the total number of spurious events in $X$ and $X^{\prime}$ respectively. SES is related to the metrics ("distances") proposed in [6]; those metrics are single numbers that quantify the synchrony between event strings. In contrast, we characterize synchrony by means of three parameters, which allows us to distinguish different types of synchrony (see, e.g., [7]). We compute those three parameters by performing inference in a probabilistic model. In the next section, we briefly describe that model, and we briefly outline two different inference algorithms.

\section{COMPUTING THE SES PARAMETERS}

We translate the above procedure to generate $X$ and $X^{\prime}$ (cf. Fig. 2) into a stochastic model. We associate to each event $X_{k}$ $(k=1, \ldots, N)$ a binary variable $B_{k}$ that equals one if $X_{k}$ is spurious and equals zero otherwise; likewise, we associate a binary variable $B_{k}^{\prime}$ to $X_{k}^{\prime}\left(k=1, \ldots, N^{\prime}\right)$. We specify the following prior distribution for the "spuriosity" string $B \triangleq$ $B_{1}, \ldots, B_{N}$ (and likewise $B^{\prime}$ ):

$$
p(b) \triangleq \prod_{k=1}^{N} p\left(b_{k}\right),
$$

where $p\left(B_{k}=1\right) \triangleq p_{\text {spur }}$ for all $k$, and $p_{\text {spur }} \in(0,1)$. The number of non-spurious events in each of the strings $X$ and $X^{\prime}$ is denoted by $M ; T_{k}$ and $T_{k}^{\prime}$ stand for the $k$-th nonspurious event in $X$ and $X^{\prime}$ respectively, where $k=1, \ldots, M$. The non-spurious events $T_{k}$ are modeled as i.i.d. random variables, distributed according to some distribution $f_{T}\left(t_{k}\right)$. In the following, we assume that $f_{T}\left(t_{k}\right) \triangleq \alpha_{T}$, where $\alpha_{T}$ is a positive real number. The event $T_{k}^{\prime}$ is related to $T_{k}$ as

$$
T_{k}^{\prime}=T_{k}+N_{k}, \quad(k=1, \ldots, M)
$$

where $N_{k}$ is an i.i.d. sequence of Gaussian random variables with mean $\delta_{T}$ and variance $\sigma_{T}^{2}$. We denote by $S_{k}$ and $S_{k}^{\prime}$ the $k$-th spurious event in $X$ and $X^{\prime}$ respectively. Similarly as $T_{k}$, the spurious events $S_{k}$ and $S_{k}^{\prime}$ are modeled as i.i.d. random variables, distributed according to some distribution $f_{S}\left(s_{k}\right)$ and $f_{S^{\prime}}\left(s_{k}^{\prime}\right)$ respectively. In the following, we assume that $f_{S}\left(s_{k}\right)=f_{S^{\prime}}\left(s_{k}^{\prime}\right) \triangleq \alpha_{S}$, where $\alpha_{S}$ is a positive real number. Note that $X$ is fully determined by $B, S$ and $T$, and likewise, $X^{\prime}$ is entirely determined by $B^{\prime}, S^{\prime}$ and $T^{\prime}$. In summary, after some straightforward algebra, we obtain the following probabilistic model:

$$
\begin{aligned}
p\left(x, x^{\prime}, t, t^{\prime}, s, s^{\prime}, b, b^{\prime} ; \delta_{T}, \sigma_{T}\right) \otimes & \beta^{N+N^{\prime}-2 M} \delta(x-g(s, t, b)) \\
\cdot & \delta\left(x^{\prime}-g\left(s^{\prime}, t^{\prime}, b^{\prime}\right)\right) \delta\left[N-M-\sum_{k=1}^{N} b_{k}\right] \\
\cdot & \delta\left[N^{\prime}-M-\sum_{k=1}^{N^{\prime}} b_{k}^{\prime}\right] \prod_{k=1}^{M} \mathcal{N}_{\delta_{t}, \sigma_{t}^{2}}\left(t_{k}^{\prime}-t_{k}\right)
\end{aligned}
$$

where $\mathcal{N}_{\mu, \sigma^{2}}$ is a Gaussian distribution with mean $\mu$ and variance $\sigma^{2}$,

$$
\beta \triangleq \frac{\alpha_{S} p_{\text {spur }}}{\sqrt{\alpha_{T}}\left(1-p_{\text {spur }}\right)}
$$

and $g$ maps the strings $B, S$ and $T$ to an event string $X$, and likewise, it maps $B^{\prime}, S^{\prime}$ and $T^{\prime}$ to an event string $X^{\prime}$. Note that $\alpha_{T}, \alpha_{S}$, and $p_{\text {spur }}$ do not need to be specified individually, since they appear in (4) only through $\beta$. The latter serves as a knob that controls the number of spurious events.

Given event strings $X$ and $X^{\prime}$, we wish to determine the parameters $\delta_{T}$ and $\sigma_{T}^{2}$, and the hidden variables $B$ and $B^{\prime}$ (from which $\rho_{\text {spur }}$ can directly be computed, cf. (1)). There are various ways to solve this inference problem. Note first of all that if $B$ and $B^{\prime}$ (and hence $T$ and $T^{\prime}$ ) are given, it is trivial to determine the maximum likelihood (ML) estimates of $\delta_{T}$ and $\sigma_{T}$. On the other hand, if $\delta_{T}$ and $\sigma_{T}$ are given, the maximum a posteriori (MAP) estimate $B^{\mathrm{MAP}}$ and $B^{\prime \mathrm{MAP}}$ of the sequences $B$ and $B^{\prime}$ respectively can be determined by applying the Viterbi algorithm on an appropriate trellis, or equivalently, by applying the max-product algorithm on a suitable factor graph [8]. Therefore, perhaps the simplest and most natural strategy to jointly estimate $B, B^{\prime}, \delta_{T}$ and $\sigma_{T}$, is to apply cyclic maximization (a.k.a "iterative conditional modes") [9]: one starts with an initial estimate $\hat{\delta}_{T}$ and $\hat{\sigma}_{T}$ of $\delta_{T}$ and $\sigma_{T}$, then, one iterates the following two steps:

1. with the current estimates $\hat{\delta}_{T}$ and $\hat{\sigma}_{T}$, determine $B$ and $B^{\prime}$ by the Viterbi/max-product algorithm,

2. with the current estimates $\hat{B}$ and $\hat{B}^{\prime}$, update the estimates $\hat{\delta}_{T}$ and $\hat{\sigma}_{T}$.

As an alternative, we have also derived an inference algorithm based on expectation maximization (EM) [10].

\section{APPLICATION TO SPIKE DATA}

We consider here in vitro intracellular electrophysiological recordings from a rat neocortical pyramidal cell [11]. The cell was stimulated with excitatory and inhibitory conductances so as to simulate synaptic inputs during neocortical sustained activity. Fig. 3(a) shows 50 trials where each time the cell was activated by the same stimulus. In order to quantify the firing 


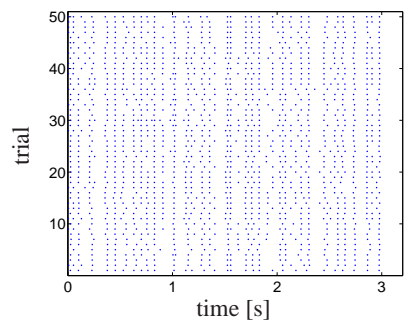

(a) Spike trains

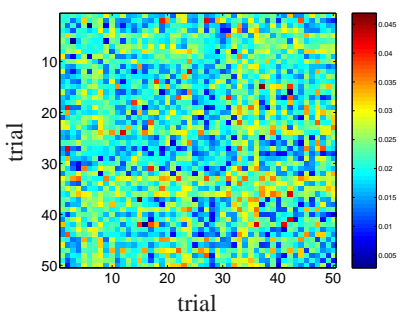

(c) $\sigma_{T}$

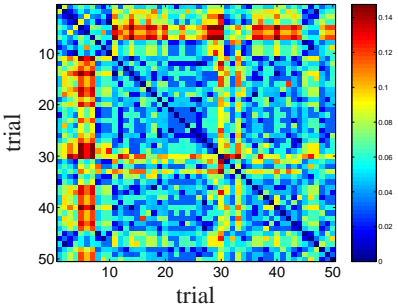

(b) $\rho_{\text {spur }}$

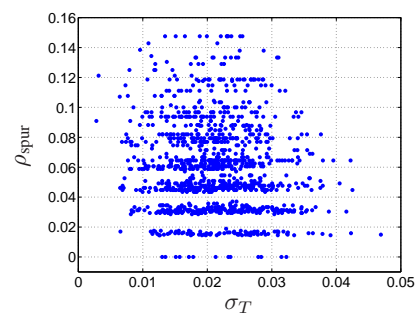

(d) $\rho_{\text {spur vs. } \sigma_{T}}$
Fig. 3. SES for in vitro intracellular recordings.

reliability of the cell [7], we computed the SES parameters $\rho_{\text {spur }}$ (see Fig. 3(b)) and $\sigma_{T}$ (see Fig. 3(c)) for each pair of trials; the offset $\delta_{T}$ is about zero, it is not relevant for the application at hand. On the average, $\rho_{\text {spur }}=6.0 \%$ and $\sigma_{T}=21 \mathrm{~ms}$. The average interspike time equals $97 \mathrm{~ms}$, which is significantly larger than $\sigma_{T}$; the firing variability of the cell can thus be considered small [7]. Fig. 3(d) show the parameters $\left(\rho_{\text {spur }}\right.$, $\sigma_{T}$ ) for each pair of trials; it can be seen from that figure that the two parameters are essentially uncorrelated. The results of Fig. 3 are only a starting point for further investigations. In the future, we plan to study for instance how the stimulus affects the SES parameters.

\section{APPLICATION TO EEG SIGNALS}

SES can be used to detect synchrony in EEG signals. Obviously, the method cannot directly be applied to EEG signals ${ }^{1}$ : the EEG signal needs to be transformed first into an event string, as we will describe in Section 5.2. We determine the SES parameters for EEG recordings from Alzheimer's disease (AD) patients and age-matched control patients; we use the SES parameters as features to classify those two types of patients. In Section 5.1, we provide information about the EEG signals we analyzed; in Section 5.3, we provide some preliminary results.

\subsection{EEG recordings}

The EEG of 23 Alzheimer disease (AD) patients and 38 agematched control patients (CTR) was recorded while in rest with closed eyes. The electrodes were located on 21 sites according to the 10-20 international system [12]. For computing the SES, we used from each recording a continuous artifact-free interval of 20s. We investigate the theta band

\footnotetext{
${ }^{1}$ We remind the reader of the important physiological difference between EEG signals and the intracellular recordings discussed in Section 4: EEG signals stem from thousands of activated neurons [12], whereas intracellular recordings measure the activation of a single neuron.
}

(3.5-7.5 Hz), since the anomalies associated with AD are the most significant in that frequency band (see [16] and references therein).

\subsection{From EEG signals to event strings}

To each EEG signal, we successively apply two transformations: (i) wavelet transform; (ii) sparsification of the wavelet representation (a.k.a. "bump modeling").

\subsubsection{Wavelet Transform}

In order to extract the oscillatory patterns in the EEG signal, we apply a wavelet transform. More specifically, we use the complex Morlet wavelets

$$
w(t)=A \exp \left(-t^{2} / 2 \sigma_{t}^{2}\right) \exp (2 i \pi f t),
$$

where $t$ is time, $f$ is frequency, $\sigma_{t}$ is a (positive) real parameter, and $A$ is a (positive) scalar normalization factor. The Morlet wavelet (6) has proven to be well suited for the timefrequency analysis of intracellular signals (see [13]). As a result, we obtain a time-frequency representation $c(f, t)$ of the EEG signal. The next transformation (i.e., sparsification) operates on the squared magnitude $z(f, t)$ of the coefficients $c(f, t)$ :

$$
z(f, t) \triangleq|c(f, t)|^{2} .
$$

\subsubsection{Sparsification}

We approximate the map $z(f, t)$ as a sum $z_{\text {bump }}(f, t, \theta)$ of a "small" number of smooth basis functions ("bumps"):

$$
z(f, t) \approx z_{\text {bump }}(f, t, \theta) \triangleq \sum_{k=1}^{N_{b}} b\left(f_{k}, t_{k}, \theta_{k}\right),
$$

where each bump $b\left(f_{k}, t_{k}, \theta_{k}\right)$ is centered at time $t_{k}$ and frequency $f_{k}$ (see Fig. 4); the vector $\theta_{k}$ contains additional bump parameters, and $\theta \triangleq\left(\theta_{1}, \theta_{2}, \ldots, \theta_{N_{b}}\right)$. The sparse bump approximation $z_{\text {bump }}(f, t, \theta)$ captures the most significant oscillatory activities in the EEG signal; we refer to [14] [15] for more information on bump modeling. For the purpose of this paper, we transform the bump train $z_{\text {bump }}(f, t, \theta)$ into a binary sequence $z_{\text {bin }}(t)$, as illustrated in Fig. 4(c). At the bump centers $t_{k}$, the sequence $z_{\text {bin }}(t)$ takes the value 1 , otherwise it equals 0 . Also other bump parameters (such as the center frequency) can be integrated in the concept of SES; this is the subject of ongoing work.

\subsection{Results and Discussion}

Our preliminary results on the classification of CTR and AD patients on the basis of synchrony are summarized in Table 1. As features, we used the SES parameters in addition to a standard synchrony measure, i.e., spectral coherence $(\mathrm{COH})$ [12]; the latter serves as a benchmark for assessing the classification performance of the SES parameters. (In the context of $\mathrm{AD}$ detection, spectral coherence has so far intensively been used for hypothesis testing: in many studies, it is verified 


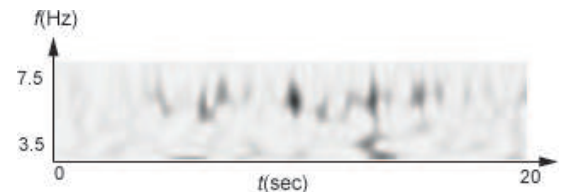

(a) Time-frequency map $z(f, t)$.

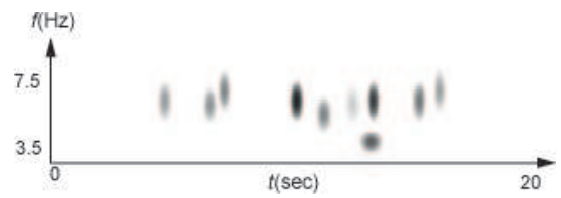

(b) Bump train approximation $z_{\text {bump }}(f, t, \theta)$.

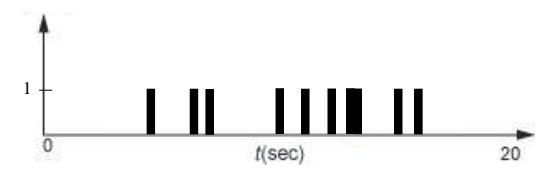

(c) Binary sequence approximation $z_{\text {bin }}(t)$.

Fig. 4. From wavelet transform to binary sequence.

whether $\mathrm{AD}$ is associated with statistically significant anomalies in the spectral coherence in certain frequency bands and cortical areas; as far as we know, spectral coherence has not yet been used to classify CTR and AD patients. As a consequence, it is hard to compare our results to previous studies.) We investigated ten different sets of features, each containing four features; with the first four sets, we test the SES parameters and coherence individually. In each of the last six sets, we combine two of those four parameters; each of the two parameters contributes two features. For all ten feature sets, we used a hyperplane as classifier ("linear discriminant analysis"); we chose this rather simple classifier (instead of a multilayer perceptron for instance) in order to avoid overfitting. We selected (by brute force optimization) the four pairs of electrodes that resulted in the highest classification performance. As can be seen from Table 1 (first four feature sets), the (individual) SES parameters $\delta_{T}$ and $\sigma_{T}$ resulted in a slightly better classification performance than coherence (in this particular experiment); of the two-parameter combinations (feature sets 5-10), the combinations $\delta_{T}-\sigma_{T}$ and $\mathrm{COH}-\rho_{\text {spur }}$ yielded the best results $(83.6 \%$ correctly classified). Moreover, we verified (similarly as in Fig. 3(d)) that the SES parameters are practically uncorrelated with coherence. The SES parameters provide thus additional information about synchrony.

Of course, we are fully aware of the fact that this simple experiment does not give the final answer. We are currently designing more involved classifiers that combine SES parameters and spectral coherence with many other features. The results of those more exhaustive experiments will be reported in the near future.

\section{CONCLUSION}

We have introduced a novel measure to quantify the synchrony between event strings, i.e., stochastic event synchrony (SES), and we have explored two applications. We believe that SES may serve as a valuable tool to study synchrony in neural sig-

\begin{tabular}{|l|c||l|c|}
\hline Features & Correct & Features & Correct \\
\hline \hline $\mathrm{COH}$ & $78.7 \%$ & $\mathrm{COH}$ and $\delta_{T}$ & $80.3 \%$ \\
\hline$\delta_{T}$ & $80.3 \%$ & $\mathrm{COH}$ and $\sigma_{T}$ & $82.0 \%$ \\
\hline$\sigma_{T}$ & $80.3 \%$ & $\mathrm{COH}$ and $\rho_{\text {spur }}$ & $83.6 \%$ \\
\hline$\rho_{\text {spur }}$ & $78.7 \%$ & $\sigma_{T}$ and $\rho_{\text {spur }}$ & $80.3 \%$ \\
\hline$\delta_{T}$ and $\sigma_{T}$ & $83.6 \%$ & $\delta_{T}$ and $\rho_{\text {spur }}$ & $80.3 \%$ \\
\hline
\end{tabular}

Table 1. Percentage of subjects correctly classified.

nals. We are currently exploring its usability in more depth, and we are developing various extensions (particularly in the context of bump modeling).

\section{ACKNOWLEDGEMENTS}

The authors wish to thank Dr. Kenji Morita from the RIKEN BSI for providing in vitro intracellular recordings (cf. Section 4). The first author is supported by a Post-Doctoral Fellowship (No. PE05060) from the Japanese Society for the Promotion of Science (JSPS).

\section{A. REFERENCES}

[1] F. Varela, J. P. Lachaux, E. Rodriguez, and J. Martinerie, "The Brainweb: Phase Synchronization and Large-Scale Integration", Nature Reviews Neuroscience, 2(4):229-39, 2001

[2] W. Singer, "Consciousness and the Binding Problem," Annals of the New York Academy of Sciences, 929:123-146, April 2001.

[3] M. Abeles, H. Bergman, E. Margalit, and E. Vaadia, "Spatiotemporal Firing Patterns in the Frontal Cortex of Behaving Monkeys," J. Neurophysiol, 70(4):16291638. 1993.

[4] S. Amari, H. Nakahara, S. Wu, and Y. Sakai, "Synchronous Firing and HigherOrder Interactions in Neuron Pool," Neural Computation, 15:127-142, 2003.

[5] H. Matsuda, "Cerebral Blood Flow and Metabolic Abnormalities in Alzheimer's Disease," Ann. Nucl. Med., vol. 15, pp. 85-92, 2001.

[6] J. D. Victor and K. P. Purpura, "Metric-space Analysis of Spike Trains: Theory, Algorithms, and Application," Network: Comput. Neural Systems, 8:17, 164, 1997.

[7] H. P. C. Robinson, "The Biophysical Basis of Firing Variability in Cortical Neurons," Chapter 6 in Computational Neuroscience: A Comprehensive Approach, Mathematical Biology \& Medicine Series, Edited By Jianfeng Feng, Chapman \& Hall/CRC, 2003.

[8] H.-A. Loeliger, "An Introduction to Factor Graphs," IEEE Signal Processing Magazine, Jan. 2004, pp. 28-41.

[9] P. Stoica and Y. Selén, "Cyclic minimizers, majorization techniques, and the expectation-maximization algorithm: a refresher," IEEE Signal Proc. Mag., January 2004, pp. 112-114.

[10] A. P. Dempster, N. M. Laird, and D. B. Rubin "Maximum Likelihood From Incomplete Data Via the EM Algorithm," Journal of the Royal Statistical Society, B 39, pp. 1-38, 1977.

[11] K. Morita and H. P. C. Robinson, unpublished.

[12] P. Nunez and R. Srinivasan, Electric Fields of the Brain: The Neurophysics of EEG, Oxford University Press, 2006.

[13] C. Tallon-Baudry, O. Bertrand, C. Delpuech, and J. Pernier, "Stimulus Specificity of Phase-Locked and Non-Phase-Locked $40 \mathrm{~Hz}$ Visual Responses in Human," Journal of Neuroscience, 1996, 16, pp. 4240-4249.

[14] F. Vialatte, Modélisation en bosses pour l'analyse des motifs oscillatoires reproductibles dans l'activité de populations neuronales : applications à l'apprentissage olfactif chez l'animal et à la détection précoce de la maladie d'Alzheimer, PhD Thesis, Paris VI University, Paris, 2005. Available from http://www.neurones.espci.fr/Theses_PS/VIALATTE_F.pdf

[15] F. Vialatte, C. Martin, R. Dubois, J. Haddad, B. Quenet, R. Gervais, and G. Dreyfus, "A Machine Learning Approach to the Analysis of Time-Frequency Maps, and Its Application to Neural Dynamics," Neural networks, 2006, in press.

[16] F. Vialatte and A. Cichocki, "Sparse Bump Sonification: a New Tool for Multichannel EEG Diagnosis of Mental Disorders; Application to the Detection of the Early Stage of Alzheimer's Disease," Proc. of ICONIP 2006, Part III, LNCS 4234 proceedings. 